Escuela de Relaciones Internacionales.

Universidad Nacional, Costa Rica.

N. 90.1 • Enero-Junio de 2017

doi: http://dx.doi.org/10.15359/ri.90-1.5

Pp. 125-140

\title{
CONDICIONES DE FINANCIAMIENTO PÚBLICO QUE ENFRENTAN LAS MICROEMPRESAS DEL SECTOR COMERCIO EN COSTA RICA
}

\author{
Cristel Avendaño Quesada* \\ María José Barrantes Barboza** \\ Paula Claro López*** \\ Daniela Solano Salazar****
}

\section{RESUMEN:}

El objetivo principal del presente trabajo es analizar las condiciones de financiamiento público que enfrentan las microempresas del sector comercio en Costa Rica. Para lo cual se analizan estudios anteriores sobre el tema y se selecciona una muestra de microempresas del sector comercio, ubicados en el cantón de Heredia. Se investiga su grado de acceso al crédito, las principales limitaciones, las instituciones y fondos que se han creado y sus respectivos requerimientos. Asimismo, el trabajo muestra como el acceso al financiamiento es clave para el crecimiento y expansión de estas empresas, por lo que el Estado debe hacer énfasis en el fomento de este sector.

Palabras claves: Financiamiento público; microempresas; sector comercio; crédito.

\begin{abstract}
:
The main objective of the present assignment is to analyze the conditions of public financing that the microenterprises of the commerce sector in Costa Rica confront. For that, we analyze previous studies on the subject and select a sample of microentrepreneurs from the commerce sector, located in Heredia's city. It investigates the degree of access to credit of microentrepreneurs, main constraints, institutions and funds that have been created, with their respective requirements. Also, the work shows how access to financing is key to the growth and expansion of these companies, so that the State should emphasize the promotion of this sector.
\end{abstract}

Keywords: Public financing; public; microenterprises; commerce sector; credit.

* Estudiante de Bachillerato en Comercio y Negocios Internacionales. Universidad Nacional de Costa Rica. Correo electrónico: crisavenque@gmail.com

** Estudiante de Bachillerato en Comercio y Negocios Internacionales. Universidad Nacional de Costa Rica. Correo electrónico: mjosebarrantes@hotmail.es

*** Estudiante de Bachillerato en Comercio y Negocios Internacionales. Universidad Nacional de Costa Rica. Correo electrónico: pau.clarol@gmail.com

**** Estudiante de Bachillerato en Comercio y Negocios Internacionales. Universidad Nacional de Costa Rica. Correo electrónico: dani95s@hotmail.com 


\section{Introducción}

Las micro, pequeñas y medianas empresas (MiPymes) ocupan un papel muy importante en la economía de Centroamérica, debido a que son fuentes de empleo e ingresos. Para Centroamérica (para efectos del trabajo entiéndase por Centroamérica a Guatemala, El Salvador, Honduras y Costa Rica), las MiPymes representan un peso importante; por ejemplo en Guatemala constituyen el $87 \%$ del empleo y cerca del 40\% del PIB, en El Salvador ocupan el 99.3\% del total de empresas activas y generan el 68\% del empleo, y en Honduras se localizan alrededor de 1.800 MiPymes afiliadas a la Cámara de Comercio. A pesar de esto, es una realidad palpable que las MiPymes enfrentan diversas restricciones en la conformación y mantenimiento de su empresa (Solís, 2016); además, en los últimos años, las microempresas han enfrentado dificultades en el acceso al sistema financiero formal (Sandi, 2015).

Para el caso de Costa Rica, se estima que para el año 2014 las MiPymes contribuyeron con el 33\% del PIB Nacional y representaban el 74.2\% del parque empresarial costarricense (MEIC, 2014). Con un avance positivo, para el 2015 las MiPymes representan el $75.5 \%$ del parque empresarial y en lo que respecta al empleo para el año 2015 las empresas medianas aportaron un 53\%, las micro un $29 \%$ y las pequeñas el 18\% (MEIC, 2016). Por lo anterior, en el país la inquietud por las MiPymes no es algo nuevo, ya que constituyen una clave importante para el progreso de Costa Rica, y es por esto que existe una ley conocida como la "Ley de fortalecimiento a las pequeñas y medianas empresas" creada con el fin de establecer los requerimientos sobre el acceso al financiamiento de las Pymes y el marco institucional (Ángulo, s. f. y MEIC, 2015).

Asimismo, en Costa Rica se han desarrollado diversos programas de ayuda para las MiPymes, como el Fondo Especial para el Desarrollo de las micro, pequeñas y medianas empresas (FODEMIPYME) administrado por el Banco Popular y el Programa de Apoyo a la Pequeña y Mediana empresa (PROPYME). Además, existen programas de apoyo a las Pymes otorgados por el Banco de Costa Rica y el Banco Nacional, que ofrecen tanto créditos como capacitaciones.

Sin embargo, la alta tasa de mortalidad de las MiPymes en Costa Rica incita a pensar que las leyes y programas creados no están cumpliendo con el objetivo. Para el 2015, cerca del $80 \%$ de las MiPymes creadas no superaba los tres años de vida, esto se debía a factores como la falta de acceso al financiamiento, imposibilidad de explotar economías de escala, la falta de habilidades de sus empleados, pocos accesos a las nuevas tecnologías, entre otras dificultades (Castillo, 2012). 
El problema reside a partir de que, en Costa Rica, el estudio sobre el acceso al crédito de las microempresas se ha dirigido principalmente a la oferta, es decir, se basan únicamente en lo que los intermediarios financieros ofrecen y no examinan lo que las empresas de las MiPymes necesitan (Díaz, 2012). Por esta razón, se proyecta abordar el tema desde la perspectiva de los dueños de microempresas del sector comercio, su panorama y sus requerimientos.

Por ende, en el siguiente trabajo se analizará el grado de acceso al crédito público de las microempresas del sector comercio en el mercado costarricense, además de indagar sobre las diversas limitaciones a las que se enfrentan estas mismas. Por tanto, se describen los diversos programas que se han creado con el fin de brindar apoyo a las MiPymes en las diferentes etapas de creación de estos, además de describir los diferentes enfoques que poseen estos programas y los diversos requisitos que deben cumplir las empresas para acceder a estos. Lo anterior, con el fin de recopilar una serie de datos que permitan conocer sobre las verdaderas razones por las cuales el crecimiento efectivo de una MiPyme se ve afectado y comprobar si la tasa de mortalidad de las Pymes en Costa Rica es tan alta durante los primeros tres años de vida (Ulloa, 2015).

El documento se organiza de acuerdo con el siguiente esquema: la primera sección aborda la justificación del tema; la segunda sección entabla la metodología utilizada para la elaboración del journal; la tercera parte muestra las características de la oferta crediticia Pyme de carácter público, análisis de las diversas instituciones públicas y fondos que se han creado para financiar a las MiPymes, los requerimientos de las entidades financieras, principales limitaciones que presentan las microempresas del sector comercio y una recopilación de datos obtenidos a través de entrevistas realizadas a pequeños comercios en el cantón de Heredia, incluyendo las implicaciones sobre el tema. Finalmente, se presentan las conclusiones y referencias de bibliografía utilizada para la elaboración del documento.

\section{Justificación}

Basados en datos del MEIC (2015), la importancia del apoyo al emprendimiento costarricense como una manera de generar crecimiento económico con empleo digno constituye una tarea prioritaria y uno de los retos asumidos por el gobierno actual de la administración Solís Rivera. Según el Plan de Desarrollo vigente 2015-2018, el empleo y la reactivación son pilares de las políticas públicas productivas. Debido a esta condición, es de suma importancia conocer las circunstancias de las MiPymes en el país durante los últimos años y las medidas que ha tomado el gobierno costarricense para mejorar y facilitar la 
creación y mantenimiento de este tipo de empresas y, por ende, comprobar su efectividad.

De acuerdo con el estado de situación de las PYME en Costa Rica, elaborado por el MEIC, para el 2015, las microempresas representaron la mayor parte de las empresas del país, las cuales contribuyeron en un $28 \%$ a generar fuentes de empleo. Asimismo, estas representaron el 27\% del total de las exportaciones realizadas por las PYMES; además, de tener un incremento de $6.7 \%$ en ventas al Estado (Cordero, 2016). Lo anterior evidencia la influencia de las microempresas en la economía del país y el potencial de estas.

Por otra parte, además de especificar y seleccionar el tamaño adecuado de la empresa, se decide determinar un sector en específico de prevalencia, que permita generar resultados de interés para el curso y para los efectos de análisis. Con base en esto, se seleccionó el sector económico comercio, ya que es el que más empleos genera, con aproximadamente 238 mil trabajadores, lo cual representa el 14\% de la fuerza laboral total del país; asimismo, dentro de la clasificación de las MiPymes por sector económico, el de comercio es el que ocupa el segundo puesto por debajo del sector de servicios con un $41 \%$ de las MiPymes del país (MEIC, 2015).

Lo anterior, según el presidente de la Cámara de Comercio, el Sr. Francisco Llobet, se debe a que usualmente el sector comercial requiere de menos inversión y conocimiento técnico, lo que coincide con un porcentaje importante de Pymes concentradas en esa actividad (La Nación, 2015). Esta característica motiva la pregunta de investigación de conocer el panorama crediticio que estas enfrentan, pues cabe la posibilidad de que la falta de conocimiento infiera en la capacidad y habilidad por la búsqueda de financiamiento.

Además, es lícito recalcar que para el año 2015 el sector comercio generó gran cantidad de empleos dentro de las MiPymes, contribuyendo con un 33\% de los empleos totales (MEIC, 2015). Sin duda alguna, este sector económico representa un pilar importante en la economía del país.

Igualmente, en lo que respecta a las exportaciones del país, el 55,6\% del valor exportado estuvo a cargo del sector comercio, que presentó una leve recuperación económica en el 2015, debido a diversas razones entre las que destacan (MEIC, 2016): Aumento de la demanda interna, apertura de nuevos centros comerciales, y la estacionalidad de las ventas en los comercios, producto de fechas especiales. Por tanto, considerando ambas partes (sector y tamaño de empresa), se obtiene que de la contribución según tamaño de empresa al valor de las exportaciones FOB (free on board) total según sector, el $42 \%$ de las microempresas se 
establecieron en el sector comercial, indicando que abarca un área importante de este sector (MEIC, 2015).

\section{Metodología}

El presente trabajo se desarrolla dentro de la temática del curso de Teorías del Comercio internacional, e incluye un análisis acerca del grado de acceso al crédito público que poseen las microempresas del sector comercio en Costa Rica. Además, intenta conocer a fondo aspectos económicos de las microempresas costarricenses y las principales limitaciones que enfrentan estas en su proceso de formación.

Asimismo, se procura conocer la oferta crediticia que el país brinda a este sector de la economía, mediante el análisis de los fondos o créditos creados por entidades financieras estatales. Por lo que, originalmente la investigación se abordará por medio del análisis de fuentes bibliográficas basadas en investigaciones previamente realizadas en temas de MiPymes, se realizará un recuento sobre las ideas presentadas en estos documentos, para, así, lograr recopilar las ideas y conclusiones más importantes que se han logrado obtener a través de los años en este tema; además, fuentes que evalúen el sector comercial y la relación de las microempresas con este.

Se utiliza un enfoque de investigación descriptivo y la técnica empleada para la obtención de los resultados se basará en una entrevista semiestructurada a sujetos microempresarios con negocios establecidos en el centro de Heredia, con el objetivo de conocer el grado de acceso al crédito que han obtenido a lo largo de los años, el conocimiento que poseen en temas de financiamiento y tramitología $\mathrm{y}$, con ello, determinar cuáles son las principales limitaciones que enfrentan o enfrentaron en la creación y consolidación de su negocio. Igualmente, se harán visitas a los principales bancos estatales del país (Banco Nacional de Costa Rica, Banco de Costa Rica, Banco Popular y de Desarrollo Comunal), con el fin de conocer de manera más detallada los requerimientos que deben cumplir las microempresas del sector comercio que deseen obtener un crédito con alguna de estas entidades. 


\section{Análisis de resultados}

\section{Características de la oferta crediticia Pyme}

La colocación de crédito durante el 2013 fue de 511.374,48 millones de colones, de los cuales 166.197.16 millones de colones se destinaron a las microempresas y 180.807,77 millones al sector comercio (ver tabla 1). Además, durante el 2013 se aprobaron 38.887 solicitudes de crédito para Pyme, de las cuales la banca pública colocó un $54.94 \%$, lo que representa una disminución de 0.22 p.p con respecto al 2012, además, la banca privada colocó un $38.99 \%$, lo que significó un aumento de 0.61p.p con respecto al 2012 (ver tabla 1) (MEIC, 2014).

\section{Tabla 1}

Colocación de crédito según tamaño de la empresa por sector económico, año 2013. Datos en millones de colones

\begin{tabular}{|l|r|r|r|r|r|c|}
\hline $\begin{array}{c}\text { Tamaño } \\
\text { de la empresa }\end{array}$ & Agricultura & Industria & Comercio & Servicios & Otros & Total \\
\hline Micro & $25.739,33$ & $6.800,83$ & $50.884,81$ & $46.684,31$ & $36.087,88$ & $166.197,16$ \\
\hline Pequeña & $11.885,94$ & $18.128,20$ & $59.411,81$ & $52.587,71$ & $24.278,68$ & $166.292,34$ \\
\hline Mediana & $10.866,46$ & $28.787,79$ & $70.511,15$ & $55.878,56$ & $12.841,02$ & $178.884,98$ \\
\hline Total & $48.491,73$ & $53.716,83$ & $180.807,77$ & $155.150,58$ & $73.207,58$ & $511.374,48$ \\
\hline
\end{tabular}

Nota: Elaboración propia con datos del MEIC, 2016.

\section{Tabla 2}

Colocación de crédito según tipo de entidad financiera, año 2013

\begin{tabular}{|l|r|r|r|r|}
\hline \multicolumn{1}{|c|}{ Institución } & \multicolumn{1}{c|}{ Micro } & \multicolumn{1}{c|}{ Pequeña } & \multicolumn{1}{c|}{ Mediana } & \multicolumn{1}{c|}{ Total } \\
\hline Banca pública & $133.173,92$ & $73.416,05$ & $75.078,40$ & $281.668,37$ \\
\hline Banca privada & $24.845,00$ & $79.663,71$ & $95.404,28$ & 199.912 .99 \\
\hline Microfinanciera & $5.329,53$ & $13.415,67$ & $6.163,60$ & $24.908,79$ \\
\hline Cooperativa & $1.175,78$ & $1.366,90$ & 334,12 & $2.876,80$ \\
\hline Fundación & $1.672,93$ & 0,00 & 0,00 & $1.672,93$ \\
\hline Fondo especial & 836,99 & 696,78 & 105,20 & $1.638,97$ \\
\hline Total general & $167.034,15$ & $168.559,11$ & $512.678,87$ & $512.678,87$ \\
\hline
\end{tabular}

Nota: Elaboración propia con datos del MEIC, 2016. 
En lo que respecta a la colocación de créditos por región, el cantón Central es el que demanda mayor cantidad de crédito con un $77 \%$ en el 2013 , cuya distribución mayor se dio en los cantones de San José (17.91\%), Escazú (17.02\%), Goicoechea (9.38\%), San Carlos (5.34\%) y Heredia (4.97\%). Lo anterior obedece a la conglomeración de empresas en este sector (ver figura 1) (MEIC, 2014).

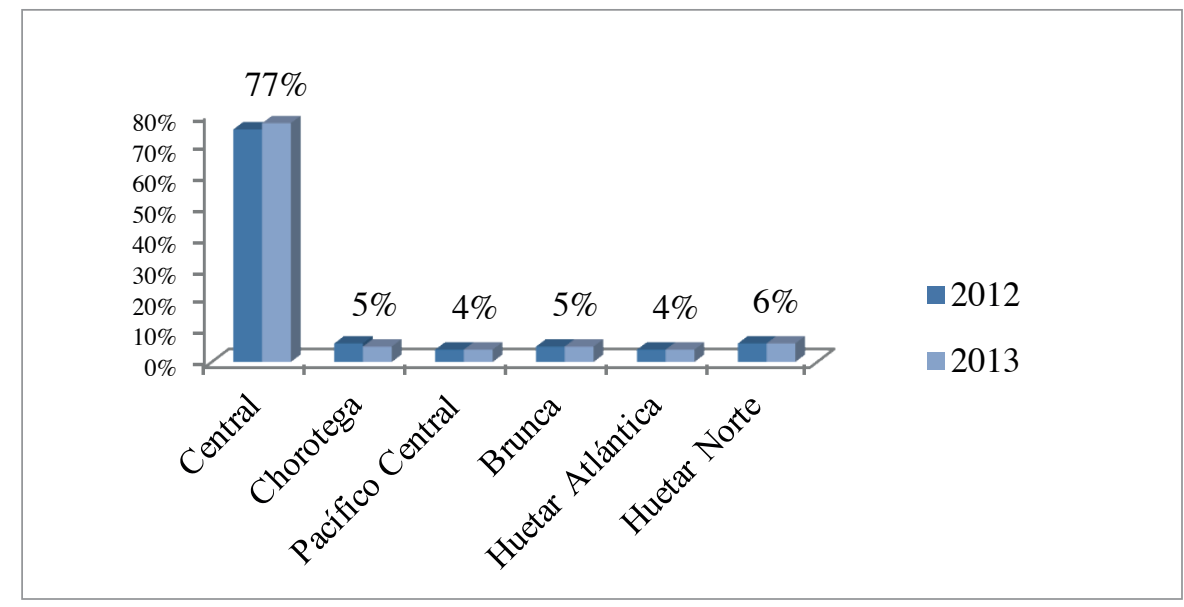

Figura 1. Distribución porcentual del crédito PYME por región, año 2012-2013. Elaboración propia con datos del MEIC, 2016.

\section{Instituciones y fondos creados para apoyar a las MiPymes}

Como parte de diversos estudios realizados, se ha concluido que el desarrollo del sector microfinanciero es clave para la generación de empleo y contribución en el PIB en países donde la actividad económica y productiva tiene un fuerte componente micro y actividades ligadas a sectores informales (Soler, Catellano, 2004). Por esta razón, el índice de inclusión financiera, mide la accesibilidad universal a productos y servicios financieros de poblaciones que siempre han estado excluidas, con el fin de estimular el crecimiento y el desarrollo económico (SIECA, 2015).

De los resultados obtenidos por el índice de inclusión financiera, en lo que respecta a los países centroamericanos, el que obtuvo mejor desempeño fue Nicaragua (51 puntos) superando escasamente a la media obtenida por el resto de países latinoamericanos. (SIECA, 2015). Costa Rica obtuvo un puntaje de 36, una de las razones principales por las que Costa Rica se encuentra con un bajo índice de inclusión financiera obedece al hecho de que la población ha tenido 
un amplio acceso a los servicios de la banca comercial, por lo que las políticas de inclusión financiera no son prioridad, por la amplia presencia de bancos públicos que han paralizado el desarrollo de un mercado para el microcrédito (SIECA, 2015).

Por lo anterior, el país ha establecido diversos programas institucionales, entre los cuales se menciona la creación en el 2008 del Sistema de Banco para el Desarrollo (SBD), en el cual se separa cierto porcentaje de los depósitos del sistema bancario para conceder créditos a sectores de bajos ingresos. Debido a esto, el sector microfinanciero, como tal, se ha visto restringido y principalmente los créditos son otorgados a través de la banca comercial, ya que se carece de normativas y definición legal sobre microcrédito y microfinanciamiento.

Asimismo, el Banco Nacional de Costa Rica y el Banco Popular y de Desarrollo Comunal ofrecen a las MiPymes créditos, además, este último tiene a disposición el Fondo para el Desarrollo de las MiPymes (FODEMIPYME). Este fue creado por la Ley 8262 y tiene como objetivo fortalecer y fomentar el desarrollo y competitividad de las MiPymes. Asimismo, cuenta con dos fondos, uno de garantías y otro de financiamiento: el primero de ellos se financia con los recursos del BPDC, provenientes del $0.25 \%$ del aporte patronal y el producto de las utilidades del mismo fondo que están exentas del impuesto sobre la renta; el segundo se conforma por el flujo anual correspondiente a un porcentaje no menor del 5\% de las utilidades del BPDC, siempre que el rendimiento de capital de esta institución supere la inflación. (Angulo).

Igualmente, se crea mediante la ley 8262 el Programa de Apoyo a la Pequeña y Mediana empresa (PROPYME), cuyo fin es financiar programas de naturaleza tecnológica que busquen mejorar la capacidad de gestión y competitividad de las MiPymes. Los recursos para este programa se obtienen directamente de los ingresos del Presupuesto Nacional. Con este programa, las MiPymes pueden recibir como máximo el $80 \%$ del costo total de los programas y proyectos aprobados, estos no pueden superar los 24 meses de duración (Angulo).

Por otra parte, el Banco de Costa Rica (BCR) ofrece el crédito MiPymes aceleración de empresas del BCR. El objetivo es que sea utilizado en inversión de activo productivo o fijo, consolidación de deudas, recuperación de capital invertido $\mathrm{u}$ otros planes de inversión aceptados por el banco; el monto mínimo que se otorga a micro y pequeñas empresas es de $\$ 1.000$, mientras que para medianas empresas es de $\$ 5.000$ y el máximo otorgado es de $\$ 1.000 .000$. Además, este banco ofrece una tarjeta de crédito para MiPymes, la cual deberá ser usada únicamente para gastos generados por el negocio; el monto mínimo otorgado es de $\$ 500$ y el máximo es de $\$ 15.000$ (BCR, 2016). 


\section{Requerimientos de las entidades financieras}

En lo que respecta a los requisitos para solicitud de crédito en entidades financieras públicas prevalecen los siguientes: Documento de identificación, documento que permita validar el domicilio de la persona solicitante, orden patronal vigente y constancia de salario, personería jurídica, certificación notarial de la distribución de la sociedad, copia del acta constitutiva, recibo de pago de impuesto a las sociedades, copia de estatutos. Además, se solicita la siguiente información financiera: certificación de ingresos por parte de un CPA, declaración de impuestos, estados financieros, balances de comprobación, detalle de ingresos y gastos, flujo de caja, detalle de las obligaciones que se atienden con los ingresos de la empresa y sus respectivos formularios.

En lo que respecta al FODEMIPYME, los requisitos que deben cumplir las MiPymes para poder acceder a este fondo son los siguientes (BPDC, 2016): a. Pago de cargas sociales, b. Cumplimiento de obligaciones tributarias, c. Cumplimiento de obligaciones laborales (pago de póliza de riesgos de trabajo), d. Cumplir con la condición de PYME, e. Permanencia en el mercado. Por otra parte, para obtener ayuda de PROPYME, las empresas deben estar atentas a la convocatoria realizada en el Diario Oficial La Gaceta (PYMES Costa Rica, 2016).

\section{Principales limitaciones de las microempresas del sector comercio}

Entre las razones por las cuales las MiPymes ven minada su capacidad, se destacan las siguientes (Castillo, 2012): Poca capacidad de administración y carencia de habilidades de su personal, imposibilidad de explotar economías de escala y poseer la información perfecta sobre oportunidades de mercado, poco acceso a las nuevas tecnologías, excesivos trámites burocráticos para su creación y operación, además, métodos débiles de organización del trabajo.

Por otra parte, los principales motivos por los que las entidades financieras y los fondos especiales rechazan las solicitudes de crédito realizadas por las MiPymes son las siguientes (MEIC, 2014): Incumplimiento de requisitos, incapacidad de pagos, malas referencias crediticias, falta de garantía, no hay un plan de inversión y la inviabilidad del proyecto.

\section{Acceso al crédito de microempresarios del sector comercio}

Del instrumento aplicado, la información obtenida indica que la edad promedio de las personas microempresarias entrevistadas se ubica en 56 años. En lo que 
respecta a la organización de la microempresa, el 70\% de los sujetos entrevistados señalaron que se identifican como persona física y el $30 \%$ restante como sociedad anónima. En cuanto al número de sujetos propietarios, la moda se ubicó en un solo propietario, estos representaron el 60\% de la muestra. Otro dato interesante es que el promedio de experiencia se ubica en 16 años, lo que indica que estas microempresas suelen perdurar con el paso del tiempo.

Por otra parte, en cuanto a la fuentes usadas para obtener el dinero para comenzar el negocio, el 58\% indicó que los fondos fueron producto de ahorros, un $23 \%$ obtuvo el dinero por medio de un prestamista informal o familiar y el $17 \%$ por un crédito bancario; cabe destacar que la mayoría de los microempresarios que solicitó un crédito posee un grado académico mayor, que quienes nunca han solicitado uno. Lo anterior, demuestra que, como fuente principal, los microempresarios se financian con ahorros propios, familiares o de personas cercanas para iniciar el negocio, lo que deja ver que el sector financiero público tiene muy poca influencia en la constitución de estas empresas.

Por otra parte, el 90\% de los sujetos entrevistados no se encuentra registrado como MyPyme por falta de información o de interés. Además, el $70 \%$ conoce sobre las tasas de interés y los requisitos para solicitar un crédito; sin embargo, el 30\% manifiesta que no es un tema de interés para la empresa, de igual manera, el $60 \%$ manifiesta no haber recibido ningún tipo de asesoría por parte de entidades públicas y privadas, el $20 \%$ recibió asesoría por parte de una entidad privada y solo el $10 \%$ por parte del Estado, lo que demuestra que el gobierno no juega un papel importante en la asesoría de estas empresas.

\section{Conclusiones}

En un país como Costa Rica, en vías de desarrollo, en el cual el dinamismo de la economía depende en su mayoría de muchas microempresas que consecuentemente generan empleo, existe un problema claro: un acceso limitado al financiamiento que no está siendo aprovechado y que implica muchos procesos y barreras administrativas que obstaculizan la evolución de estas. Este aspecto, además, limita la oportunidad de incrementar inversiones en recurso humano, mejoramiento técnico, tecnología, infraestructura y demás por parte de las empresas, pues, indudablemente, el financiamiento es necesario para una empresa que no tenga otra fuente de inversión.

Como resultado de la investigación realizada por medio del análisis de datos provenientes de fuentes consultadas y con el trabajo de campo realizado, es posible indicar que, a pesar de la oferta crediticia que tiene la banca estatal para 
las MiPymes. como consecuencia de lo complicado que resultan los requerimientos, las microempresas han optado por constituirse por medio de ahorros o de préstamos de fuentes informales, como familiares, amigos y prestamistas, por lo que su acceso al crédito suele ser muy poco en cuanto a créditos formales.

De acuerdo con las estadísticas, se concluye que existe un problema de estructura financiera en Costa Rica, pues el financiamiento no se ajusta a la realidad actual que implica un movimiento y cambio constante global, en donde se requiere adaptación eficiente y rápida. Un ejemplo es el reducido apoyo financiero que tienen los grupos emprendedores, pues en la mayoría de las entidades financieras uno de los requisitos para acceder al crédito es que la empresa lleve alrededor de dos años funcionando.

Conjuntamente, si bien el gobierno actual afirma que el emprendimiento y el sector de las MiPymes han sido una prioridad en estos años, pareciera ser que las estrategias no están siendo del todo efectivas, pues no se evidencia un aumento de microempresas que adquieren financiamiento público, debido a las razones de exigencia de garantías y requerimientos que los entes microempresarios no poseen: altas tasas de interés que ahuyentan al empresario y falta de ingresos. Por esto, las políticas de apoyo bien diseñadas y ejecutadas pueden mejorar el desempeño de las microempresas dentro del parque empresarial.

\section{Recomendaciones}

Se recomienda al Gobierno no insistir en la aprobación de nuevos impuestos, ya que ello afectaría la confianza del sector privado y las expectativas de contratación e inversión del sector comercial. Asimismo, el sector comercio es el que más empleo genera en la economía costarricense, por lo tanto, se deberían crear fondos o créditos especiales adaptados a las cualidades actuales de este sector para fortalecer sus empresas.

Además, es necesario reestructurar los procesos y requisitos que enfrentan los entes microempresarios a la hora de solicitar un crédito, tomando en cuenta sus características y necesidades. Por otra parte, ofrecer más capacitaciones, cursos o talleres con el fin de motivar el crecimiento y desarrollo de las microempresas. También, instruir a los dueños y dueñas de MiPymes en temas administrativos, tecnológicos y financieros; así como brindar asesoramiento especial a microempresas que deseen exportar sus productos.

Implementar cursos de emprendedurismo en escuelas y colegios, con el fin de desarrollar habilidades y fortalecer la formación de nuevas generaciones, de 
manera que sea posible la creación de microempresas capaces de ofrecer productos innovadores y con un alto valor agregado. El tema de la capacitación constante, enfocada en certificación de calidad, recurso humano y ambiente, no debe dejarse de lado, pues la generación de nuevos conocimientos permite realizar cambios estructurales que potencializan y sostienen las empresas dentro del mercado.

Por último, fortalecer los encadenamientos entre las microempresas, mediante alianzas entre los diferentes negocios o redes empresariales, con el propósito de que las empresas amplíen y logren permanencia en el mercado, y mejoren en bloque su posición competitiva.

\section{Implicaciones}

Costa Rica debería hacer un esfuerzo por disminuir los procesos burocráticos que se requieren para iniciar y mantener en operación un negocio, ya que si esto no se realiza, los grupos microempresarios del país no se van a preocupar por formalizar sus empresas, lo que causaría que la informalidad de estas siga aumentando. Lo anterior afecta, de manera negativa, la economía del país, pues se interrumpe el ciclo económico, debido a que estas empresas no estarían aportando impuestos de manera directa a los diferentes entes estatales.

Asimismo, Costa Rica debe fijar atención en la promoción de las MiPymes, ya que estas empresas son las que generan la mayor parte de los empleos del país, además, de que ocupan un porcentaje considerable en el producto interno bruto. Si lo anterior no se hace, causaría que este sector se torne poco atractivo para nuevos grupos empresarios, y que los que ya se encuentran se vean afectados por el poco apoyo que se les brinda. Además, si se añade el hecho de que muchas MiPymes se dedican al sector comercial, ello representa una gran cantidad de oportunidades en materia de inversión, expansión y posicionamiento que se estarían desaprovechando.

Por lo anterior, el país debería trabajar en la implementación de mayores opciones de financiamiento para estas empresas, que estas mismas se adapten a las necesidades de los grupos microempresarios del país y no solo se enfoquen en lo que las entidades financieras ofrecen. Analizando el panorama, la falta de financiamiento reduce la competitividad de las empresas y su habilidad para ganar campo en nuevas oportunidades de negocio, pues se requiere de inversión para crecer, y la falta de este recurso se traduce en la incapacidad de poder expandir la producción, lo cual bloquea la evolución de las empresas. 
Lo expuesto anteriormente afecta, de manera negativa, el empleo del país, ya que como se muestra en los datos presentados a lo largo del documento, la habilidad de generar empleo en Costa Rica depende, en su gran mayoría, de pequeñas empresas, las cuales, a través del tiempo, se expanden potencialmente y brindan oportunidades de sobrevivencia al fragmento más pobre del país, por lo que el cierre de estas implicaría un aumento en la problemática social de Costa Rica. Igualmente, se debe trabajar en la mejora del sistema financiero público, dotar tanto a los grupos microempresarios como a las entidades financieras de nuevas herramientas que les permitan a las MiPymes obtener un acceso más directo al crédito.

Por último, el gobierno debe implementar más programas de asesoramiento para los grupos microempresarios del país, donde se les guíe en la administración de sus negocios y su personal, además, se les brinden herramientas que faciliten el acceso a las nuevas tecnologías. Esto, debido a que en la actualidad, la falta de conocimiento en temas como manejo de personal y finanzas constituyen una de las principales limitaciones de las MiPymes, lo cual se evidencia en los sujetos microempresarios entrevistados, ya que solo el 10\% utilizaba estados financieros para el control de las cuentas del negocio, mientras que el $90 \%$ restantes empleaban las anotaciones escritas. Con los apoyos adecuados, el escenario podría mejorar y las políticas planteadas se verán plasmadas en los resultados, activando la participación de las microempresas en las oportunidades de financiamiento. En conclusión, el fortalecimiento general de las MiPymes, impulsado por un mayor financiamiento, tendría un efecto directo en el desarrollo del país. 


\section{Referencias}

Angulo, J. E. (s. f). Informe final: PYME y Banca de Desarrollo. San José. Recuperado de http://www.estadonacion.or.cr/files/biblioteca_virtual/011/Pyme Banca_Desarrollo.pdf

Acuerdo SUGEF 1-05 (2014). Reglamento para la calificación de deudores. Recuperado de http://www.sugef.fi.cr/normativa/normativa_vigente/documentos/ SUGEF\%201-05\%20(v44\%20junio\%202014).pdf

Aymerich, D. (s. f). Sistema de Banca para Desarrollo permite apertura de GRAMEEN en Turrialba. Ministerio de Economía Industria y Comercio. Recuperado de http://www.meic.go.cr/comunicado/311/sistema-de-banca-paradesarrollo-permite-apertura-de-grameen-en-turrialba

Banco de Costa Rica. (2016). Crédito MiPymes aceleración de empresas micro, pequeña y mediana empresa. Autor.

Banco de Costa Rica. (2016). Solicitud de crédito micro, pequeña y mediana empresa. Autor.

Banco Popular y de Desarrollo Comunal (BPDC). (2016). Requisitos crédito persona física y persona jurídica. Autor.

Banco Popular y de Desarrollo Comunal (BPDC). (2016). Fondo para el desarrollo de las micro y pequeñas empresas (FODEMIPYME). Recuperado de http:// si.cultura.cr/financiamiento/banco-popular-y-de-desarrollo-comunal-fondopara-el-desarrollo-de-las-micro-pequenas

Brenes, L. (2009). Estado nacional de las micro, pequeñas y medianas empresas formales. Costa Rica: EUNED.

Cámara de Comercio de Costa Rica. (2016). Índice de confianza del sector comercial 2015. Recuperado de http://camara-comercio.com/wp-content/ uploads/2016/02/icscDic2015.pdf

Castillo, D. (2012). Acceso a financiamiento y falta de capacidad minan a las mipymes. crhoy.com. Recuperado de http://www.crhoy.com/acceso-a-financiamiento-y-falta-de-capacidad-minan-a-las-mipymes/

Cordero, C. (2016). Pymes aumentan participación en exportaciones y ventas al Estado. La Nación. Recuperado de http://www.nacion.com/economia/empresa$\underline{\text { rial/Pymes-aumentaron-exportaciones-ventas } 0 \text { 1555444516.html }}$

Crhoy.com. (2014). La falta de financiación y la poca innovación, problemas de las PYMES en América Latina. Recuperado de http://www.crhoy.com/lafaltadefinanciacionylapocainnovacionproblemas-delaspymesenamericalatina/ 
Díaz, E. M. (2012). El acceso al crédito para microempresas en Alajuela, Cartago y Heredia. Revista de Ciencias Económicas de la UCR, 1-26. Recuperado de http://revistas.ucr.ac.cr/index.php/economicas/article/viewFile/8050/7664

Fallas, C. (2016). Encadenamientos fortalecen los negocios artesanales en Costa Rica. El Financiero. Recuperado de http://www.elfinancierocr.com $/ \mathrm{m} / \mathrm{nego-}$ cios/productos artesanales-La_Ventanita_Meraki-Cafe_Miel-Manos en la_Masa-Arbol_de_Seda_0_947905222.html

Fernández, E. (2016). Sector comercio de Costa Rica está menos confiado, pero mantiene optimismo. El Financiero. Recuperado de http://www.elfinancierocr.com/negocios/Sector-comercial-presenta-confianza-economica $0 \quad 934106582 . \mathrm{html}$

Grameen Costa Rica. (2014). Título de lo que consultó. Recuperado de http://www. grameencostarica.com

Ministerio de Economía, Industria y Comercio. (2014). Pymes impactan el 33\% del PIB. Recuperado de http://www.meic.go.cr/comunicado/387/pymes-impactan-en-el-33-del-pib

Ley N. ${ }^{\circ}$ 8262. Ley de fortalecimiento de las pequeñas y medianas empresas y sus reformas. (2002). Recuperado de http://costarica.eregulations.org/media/ Ley $\% 208262 \% 20$ PYME.PDF

Ministerio de Economía, Industria y Comercio (MEIC). (2014). Estudio sobre la caracterización de la oferta financiera y no financiera en apoyo a las PYME en 2013. Recuperado de http://www.pyme.go.cr/media/archivo/menul/estudiocreditopyme13.pdf

Ministerio de Economía, Industria y Comercio (MEIC). (2016). Estado de situación de las Pymes en Costa Rica. Recuperado de http://reventazon.meic. go.cr/informacion/estudios/2016/pymes/informe.pdf

Nelson, J. (2014). Crédito comunal: Una opción de financiamiento para los microempresarios. El Financiero. Recuperado de http://www.elfinancierocr.com/pymes/credito comunal-pymes-micro_empresas-financiamiento $0 \quad 595740427 . \mathrm{html}$

Ortega, K. (2016). Conozca varias opciones financieras para que su negocio crezca. El Financiero. Recuperado de http://www.elfinancierocr.com/pymes/Conozca-opciones-financieras-negocio-crezca 0 955104489.html

Reglamento General a la Ley No. 8262 de Fortalecimiento de las pequeñas y medianas empresas. (2006). Recuperado de http://www.mag.go.cr/legislacion/2006/de-33111.pdf 
Rodríguez, R. (2016). Más de 100 pymes le esperan para crear sinergias. La República. Recuperado de https://www.larepublica.net/noticia/mas de 100 pymes le esperan_para crear_sinergias/

Sandí, V. (2015). Microenterprises access to microfinance in the services sector in Malaysia and Costa Rica: an institutional perspective (Tesis de doctorado). Universidad Kebangsaam Malaysia.

SIECA. (2015). La importancia del sector micro financiero en Centroamérica. Recuperado de http://www.sieca.int/PortalData/Documentos/CDFC00AE8666-4DA5-B7FF-2E669558B8E3.pdf

Solís, L. (2016). Las MIPYMES se están convirtiendo en el motor económico de la economía en Centroamérica. Aun así, enfrentan retos importantes, en especial, el financiamiento. Revista Summa. Recuperado de http://www.revistasumma.com/diagnostico-de-las-pymes-en-centroamerica/

Titelman, D. (2011). Cepal. Financiamiento en Costa Rica: micro, pequeña y mediana empresa. Qué nos dice la demanda? Recuperado de http://www. elfinancierocr.com/biblioteca/Financiamiento-RicamicropequenaTitelmanEstudiosDesarrollo-Cepal2011_ELFFIL20130731_0019.pdf

Ulloa, J. N. (2015). La 'muerte’ de las pymes: ¿Cuánto tiempo duran los pequeños negocios y por qué? El Financiero. Recuperado de http://www.elfinancierocr.com/pymes/mortalidad-muerte-pymes-empresas-desaparicion-emprendimiento_0_699530049.html

Viloria, E. (2013). Los microcréditos. El Financiero. Recuperado de http://www. elfinancierocr.com/opinion/Opinion-Eduardo_Viloria-microcreditos-banca_de_desarrollo_0_233976648.html 